\title{
Closed-Form Solutions for a Nonlinear Partial Differential Equation Arising in the Study of a Fourth Grade Fluid Model
}

\author{
Taha Aziz and F. M. Mahomed \\ Centre for Differential Equations, Continuum Mechanics and Applications, School of Computational and \\ Applied Mathematics, University of the Witwatersrand, Wits 2050, South Africa \\ Correspondence should be addressed to Taha Aziz, tahaaziz77@yahoo.com
}

Received 27 July 2012; Accepted 10 September 2012

Academic Editor: Mehmet Pakdemirli

Copyright ( 2012 T. Aziz and F. M. Mahomed. This is an open access article distributed under the Creative Commons Attribution License, which permits unrestricted use, distribution, and reproduction in any medium, provided the original work is properly cited.

\begin{abstract}
The unsteady unidirectional flow of an incompressible fourth grade fluid bounded by a suddenly moved rigid plate is studied. The governing nonlinear higher order partial differential equation for this flow in a semiinfinite domain is modelled. Translational symmetries in variables $t$ and $y$ are employed to construct two different classes of closed-form travelling wave solutions of the model equation. A conditional symmetry solution of the model equation is also obtained. The physical behavior and the properties of various interesting flow parameters on the structure of the velocity are presented and discussed. In particular, the significance of the rheological effects are mentioned.
\end{abstract}

\section{Introduction}

There has been substantial progress in the study of the behavior and properties of viscoelastic fluids over the past couple of years. This progress is due to the fact that these viscoelastic materials are involved in many manufacturing processes in today's industry. Modelling viscoelastic flows is important for understanding and predicting the behavior of processes and thus for designing optimal flow configurations and for selecting operating conditions. Most of the viscoelastic fluids in industry do not adhere to the commonly accepted assumption of a linear relationship between the stress and the rate of strain and thus are characterized as non-Newtonian fluids. As a result of their complex physical structure and diversity in nature, these materials cannot have a single constitutive relation which describes all the properties of viscoelastic fluids. The flow properties of viscoelastic (non-Newtonian) fluids are quite different from those of viscous and Newtonian fluids. Therefore, in practical applications, one cannot replace the behavior of non-Newtonian fluids with Newtonian fluids 
and it is very important to analyze the flow behavior of non-Newtonian fluids in order to obtain a thorough understanding and to improve the utilization in various manufactures.

The most interesting and important task that we need to address when dealing with the flow problems of non-Newtonian fluids is that the governing equations of these models are of higher order and much more complicated as compared with Navier-Stokes equations. Such fluids have been modelled by constitutive equations which vary greatly in complexity. The resulting nonlinear equations are not easy to solve analytically. However, various researchers [1-11] in the field have recently engaged themselves in finding analytical solutions for such flow problems.

To date, very little attention has been given on the flows of fourth grade fluids $[12,13]$. This model is the most generalized model among the differential type fluids. The fourth grade fluid model is known to capture most of the non-Newtonian flow properties at one time. This model is known to have interesting non-Newtonian flow properties such as shear thinning and shear thickening that many other non-Newtonian models do not exhibit. This model is also capable of predicting the normal stress effects that lead to phenomena like "die-swell" and "rod-climbing" [14]. With these facts in mind, we have considered a fourth grade fluid model in this study. In general, the governing equations for the flow problems of fourth grade fluids are up to fifth-order nonlinear equations. Literature survey shows that very limited studies are reported and these investigations further narrow down when we speak about closed-form solutions of these problems. However, there are few investigations available in the literature in which researchers have utilized various approaches to construct solutions of fourth grade fluid flow problems. For example, [15] Wang and Wu have tackled the problem for the unsteady flow of fourth grade fluid due to an oscillating plate using numerical methods. Recently, Siddiqui et al. [16] have obtained an optimal homotopy type solution for the thin film flow of a fourth grade fluid down a vertical cylinder by using the homotopy perturbation method (HPM). Hayat et al. [17-20] studied the fourth grade fluid problems in different types of flow situations by using the homotopy analysis method (HAM). The steady flow of a fourth grade fluid past a porous plate was treated by Marinca et al. [21] with the help of the optimal homotopy asymptotic method (OHAM). Despite all of these works in recent years, the exact closed-form solutions for the problems dealing with the flow of fourth grade fluids are still rare in the literature.

Lie's theory of differential equations (see $[22,23]$ ) was inaugurated and utilized in the solution of differential equations by the Norwegian mathematician Marius Sophus Lie in the 1870s. The main motive of the Lie symmetry analysis formulated by Lie is to find one or several parameters local continuous transformation groups leaving the equations invariant. Thus, the symmetry method for differential equations provide a rigorous way to classify them according to their invariance properties. This allows us to obtain group invariant and partially invariant solutions of differential equations in a tractable manner. The Lie symmetry approach has thus become of great importance in the theory and applications of differential equations and widely applied by several authors to solve difficult nonlinear problems particularly dealing with the flows of non-Newtonian fluids [24-28]. The conditional symmetry approach or what is called the nonclassical symmetry approach, which is an extension of the Lie approach, was originated in the work of Bluman and Cole [29]. There are equations arising in applications that do not admit Lie point symmetries but have conditional symmetries. Thus, this method is also powerful in obtaining exact solutions of such problems. In recent years, interest in the conditional symmetry approach has intensified. This method has been used successfully to obtain new exact solutions for a number of interesting nonlinear PDEs [30-32]. 
Motivated by the above-mentioned analysis, the aim of the present work is to venture further in the regime of fourth grade fluid. We investigate the time-dependent flow of an incompressible fourth grade fluid over a flat rigid plate. The motion is caused due to the motion of the plate in its own plane ( $x z$-plane) with an arbitrary velocity $V(t)$. Reductions and exact solutions of the governing nonlinear PDE for the unidirectional flow of a fourth grade fluid are established using the classical and the conditional symmetry approaches. Finally, the influence of physically applicable parameters of the flow model are studied through several graphs and appropriate conclusions are drawn.

\section{Fundamental Equations}

The basic equations governing the time-dependent flow of an incompressible fluid are the continuity equation and the momentum equation, namely,

$$
\operatorname{div} \mathbf{V}=\mathbf{0}, \quad \rho \frac{d \mathbf{V}}{d t}=\operatorname{div} \mathbf{T},
$$

where $\mathbf{V}$ is the velocity vector, $\rho$ the density of the fluid, $d / d t$ the material time derivative, and $\mathrm{T}$ the Cauchy stress tensor. For a fourth grade fluid, the Cauchy stress tensor satisfies the constitutive equations $[17,18]$

$$
\mathbf{T}=-p \mathbf{I}+\sum_{j=1}^{n} \mathbf{S}_{j} \quad \text { with } n=4
$$

where $p$ is the pressure, $\mathbf{I}$ the identity tensor, and $\mathbf{S}_{j}$ the extra stress tensor

$$
\begin{gathered}
\mathbf{S}_{1}=\mu \mathbf{A}_{1}, \\
\mathbf{S}_{2}=\alpha_{1} \mathbf{A}_{2}+\alpha_{2} \mathbf{A}_{1}^{2}, \\
\mathbf{S}_{3}=\beta_{1} \mathbf{A}_{3}+\beta_{2}\left(\mathbf{A}_{1} \mathbf{A}_{2}+\mathbf{A}_{2} \mathbf{A}_{1}\right)+\beta_{3}\left(\operatorname{tr} \mathbf{A}_{1}^{2}\right) \mathbf{A}_{1} \\
\mathbf{S}_{4}=\gamma_{1} \mathbf{A}_{4}+\gamma_{2}\left(\mathbf{A}_{3} \mathbf{A}_{1}+\mathbf{A}_{1} \mathbf{A}_{3}\right)+\gamma_{3} \mathbf{A}_{2}^{2}+\gamma_{4}\left(\mathbf{A}_{2} \mathbf{A}_{1}^{2}+\mathbf{A}_{1}^{2} \mathbf{A}_{2}\right) \\
+\gamma_{5}\left(\operatorname{tr} \mathbf{A}_{2}\right) \mathbf{A}_{2}+\gamma_{6}\left(\operatorname{tr} \mathbf{A}_{2}\right) \mathbf{A}_{1}^{2}+\left[\gamma_{7} \operatorname{tr} \mathbf{A}_{3}+\gamma_{8} \operatorname{tr}\left(\mathbf{A}_{2} \mathbf{A}_{1}\right)\right] \mathbf{A}_{1} .
\end{gathered}
$$

Here $\mu$ is the dynamic viscosity, $\alpha_{i}(i=1,2), \beta_{i}(i=1,2,3)$, and $\gamma_{i}(i=1,2, \ldots, 8)$ are material constants. The Kinematical tensors $\mathbf{A}_{1}$ to $\mathbf{A}_{4}$ are the Rivlin-Ericksen tensors defined by

$$
\begin{gathered}
\mathbf{A}_{1}=(\operatorname{grad} \mathbf{V})+(\operatorname{grad} \mathbf{V})^{T} \\
\mathbf{A}_{n}=\frac{d \mathbf{A}_{n-1}}{d t}+\mathbf{A}_{n-1}(\operatorname{grad} \mathbf{V})+(\operatorname{grad} \mathbf{V})^{T} \mathbf{A}_{n-1} ; \quad(n>1)
\end{gathered}
$$

in which grad is the gradient operator. 


\section{Flow Development}

Let an infinite rigid plate occupy the plane $y=0$ and a fourth grade fluid the half-space $y>0$. The $x$-axis and $y$-axis are chosen parallel and perpendicular to the plate. For $t>0$, the plate moves in its own plane with arbitrary velocity $V(t)$. By taking the velocity field $(u(y, t), 0,0)$, the conservation of mass equation is identically satisfied. The governing PDE in $u$ is obtained by substituting (2.2)-(2.5) into (2.1) and rearranging. We deduce the following governing equation in the absence of the modified pressure gradient:

$$
\begin{aligned}
\rho \frac{\partial u}{\partial t}= & \mu \frac{\partial^{2} u}{\partial y^{2}}+\alpha_{1} \frac{\partial^{3} u}{\partial y^{2} \partial t}+\beta_{1} \frac{\partial^{4} u}{\partial y^{2} \partial t^{2}}+6\left(\beta_{2}+\beta_{3}\right)\left(\frac{\partial u}{\partial y}\right)^{2} \frac{\partial^{2} u}{\partial y^{2}} \\
& +\gamma_{1} \frac{\partial^{5} u}{\partial y^{2} \partial t^{3}}+\left(6 \gamma_{2}+2 \gamma_{3}+2 \gamma_{4}+2 \gamma_{5}+6 \gamma_{7}+2 \gamma_{8}\right) \frac{\partial}{\partial y}\left[\left(\frac{\partial u}{\partial y}\right)^{2} \frac{\partial^{2} u}{\partial y \partial t}\right] .
\end{aligned}
$$

The above equation is subject to the following boundary and initial conditions:

$$
\begin{gathered}
u(0, t)=U_{0} V(t), \quad t>0, \\
u(\infty, t)=0, \quad t>0, \\
u(y, 0)=I(y), \quad y>0, \\
\frac{\partial u(y, 0)}{\partial t}=J(y), \quad y>0, \\
\frac{\partial^{2} u(y, 0)}{\partial t^{2}}=K(y), \quad y>0,
\end{gathered}
$$

where $U_{0}$ is the reference velocity and $V(t), I(y), J(y), K(y)$ are the unspecified functions. The first boundary condition (3.2) is the no-slip condition and the second boundary condition (3.3) says that the main stream velocity is zero. This is not a restrictive assumption since we can always measure velocity relative to the main stream. The initial condition (3.4) indicates that initially the fluid is moving with some nonuniform velocity $I(y)$. The remaining two initial conditions are the extra two conditions imposed to make the problem well posed.

We define the dimensionless parameters as

$$
\begin{gathered}
\bar{u}=\frac{u}{U_{0}}, \quad \bar{y}=\frac{U_{0} y}{v}, \quad \bar{t}=\frac{U_{0}^{2} t}{v}, \quad \bar{\alpha}=\frac{\alpha_{1} U_{0}^{2}}{\rho v^{2}}, \quad \bar{\beta}_{1}=\frac{\beta_{1} U_{0}^{4}}{\rho v^{3}}, \quad \bar{\beta}=\frac{6\left(\beta_{2}+\beta_{3}\right) U_{0}^{4}}{\rho v^{3}}, \\
\bar{\gamma}_{1}=\frac{\gamma_{1} U_{0}^{6}}{\rho v^{4}}, \quad \bar{\gamma}=\left(3 \gamma_{2}+\gamma_{3}+\gamma_{4}+\gamma_{5}+3 \gamma_{7}+\gamma_{8}\right) \frac{U_{0}^{6}}{\rho v^{4}} .
\end{gathered}
$$


Under these transformations, the governing (3.1) and the corresponding initial and the boundary conditions (3.2)-(3.6) take the form

$$
\begin{gathered}
\frac{\partial u}{\partial t}=\frac{\partial^{2} u}{\partial y^{2}}+\alpha \frac{\partial^{3} u}{\partial y^{2} \partial t}+\beta_{1} \frac{\partial^{4} u}{\partial y^{2} \partial t^{2}}+\beta\left(\frac{\partial u}{\partial y}\right)^{2} \frac{\partial^{2} u}{\partial y^{2}}+\gamma_{1} \frac{\partial^{5} u}{\partial y^{2} \partial t^{3}}+2 \gamma \frac{\partial}{\partial y}\left[\left(\frac{\partial u}{\partial y}\right)^{2} \frac{\partial^{2} u}{\partial y \partial t}\right], \\
u(0, t)=V(t), \quad t>0, \\
u(\infty, t)=0, \quad t>0, \\
u(y, 0)=f(y), \quad y>0, \\
\frac{\partial u(y, 0)}{\partial t}=g(y), \quad y>0, \\
\frac{\partial^{2} u(y, 0)}{\partial t^{2}}=h(y), \quad y>0,
\end{gathered}
$$

where $f(y)=I(y) / U_{0}, g(y)=J(y) / U_{0}$ and $h(y)=K(y) / U_{0}$. The functions $V(t), f(y), g(y)$, and $h(y)$ are as yet arbitrary. These functions are constrained in the next section when we seek closed-form solutions using the symmetry technique. For simplicity, we have neglected the bars in all the nondimensional quantities. We consider classical and conditional symmetries of (3.8). Equation (3.8) only admits translational symmetries in variables $t, y$, and $u$; thus, the travelling wave solutions are investigated in Section 4 . In Section 5, we provide a conditional symmetry solution as well.

\section{Travelling Wave Solutions}

Travelling wave solutions are special kinds of group invariant solutions which are invariant under a linear combination of time-translation and space-translation symmetry generators. It can easily be seen that (3.8) admits Lie's point symmetry generators, $\partial / \partial t$ (time-translation) and $\partial / \partial y$ (space-translation in $y$ ), so that we can construct travelling wave solutions for the model equation.

\subsection{Backward Wave-Front Type Travelling Wave Solutions}

Let $X_{1}$ and $X_{2}$ be time-translation and space-translation symmetry generators, respectively. Then the solution corresponding to the generator

$$
X=X_{1}-c X_{2}, \quad(c>0)
$$

would represent backward wave-front type travelling wave solutions. In this case, the waves are propagating towards the plate. The Lagrangian system corresponding to (4.1) is

$$
\frac{d y}{-c}=\frac{d t}{1}=\frac{d u}{0} .
$$


Solving (4.2), invariant solutions are given by

$$
u(y, t)=F(\xi) \quad \text { with } \xi=y+c t
$$

Making use of (4.3) in (3.8) results in a fifth-order ordinary differential for $F(\xi)$

$$
c \frac{d F}{d \xi}=\frac{d^{2} F}{d \xi^{2}}+\alpha c \frac{d^{3} F}{d \xi^{3}}+\beta_{1} c^{2} \frac{d^{4} F}{d \xi^{4}}+\beta\left(\frac{d F}{d \xi}\right)^{2} \frac{d^{2} F}{d \xi^{2}}+\gamma_{1} c^{3} \frac{d^{5} F}{d \xi^{5}}+2 \gamma \frac{d}{d \xi}\left[c\left(\frac{d F}{d \xi}\right)^{2} \frac{d^{2} F}{d \xi^{2}}\right] .
$$

Thus, the PDE (3.8) became an ODE (4.4) along certain curves in the $y-t$ plane. These curves are called characteristic curves or just the characteristics. In order to solve (4.4) for $F(\xi)$, we assume a solution of the form

$$
F(\xi)=A \exp (B \xi)
$$

where $A$ and $B$ are the constants to be determined. Inserting (4.5) in (4.4) we obtain

$$
\left(-c B+B^{2}+c \alpha B^{3}+\beta_{1} c^{2} B^{4}+\gamma c^{3} B^{5}\right)+e^{2 B \xi}\left(\beta A^{2} B^{4}+6 c \gamma A^{2} B^{5}\right)=0 .
$$

Separating (4.6) in powers of $e^{0}$ and $e^{2 B \xi}$, we find

$$
\begin{gathered}
e^{0}:-c B+B^{2}+c \alpha B^{3}+\beta_{1} c^{2} B^{4}+\gamma c^{3} B^{5}=0 \\
e^{2 B \xi}: \beta A^{2} B^{4}+6 c \gamma A^{2} B^{5}=0 .
\end{gathered}
$$

From (4.8), we deduce

$$
B=\frac{-\beta}{6 c \gamma}
$$

Using the value of $B$ in (4.7), we obtain

$$
\frac{\beta}{6 \gamma}+\frac{\beta^{2}}{(6 c \gamma)^{2}}-\frac{\beta^{3} \alpha c}{(6 c \gamma)^{3}}+\frac{\beta_{1} \beta^{4} c^{2}}{(6 c \gamma)^{4}}-\frac{\gamma \beta^{5} c^{3}}{(6 c \gamma)^{5}}=0
$$

Thus, the exact solution for $F(\xi)$ (provided the condition (4.10) holds) can be written as

$$
F(\xi)=A \exp \left[\frac{-\beta \xi}{6 c r}\right] .
$$

So the exact solution $u(y, t)$ which satisfies the condition (4.10) is

$$
u(y, t)=\exp \left[\frac{-\beta(y+c t)}{6 c r}\right] \text { with } c>0
$$




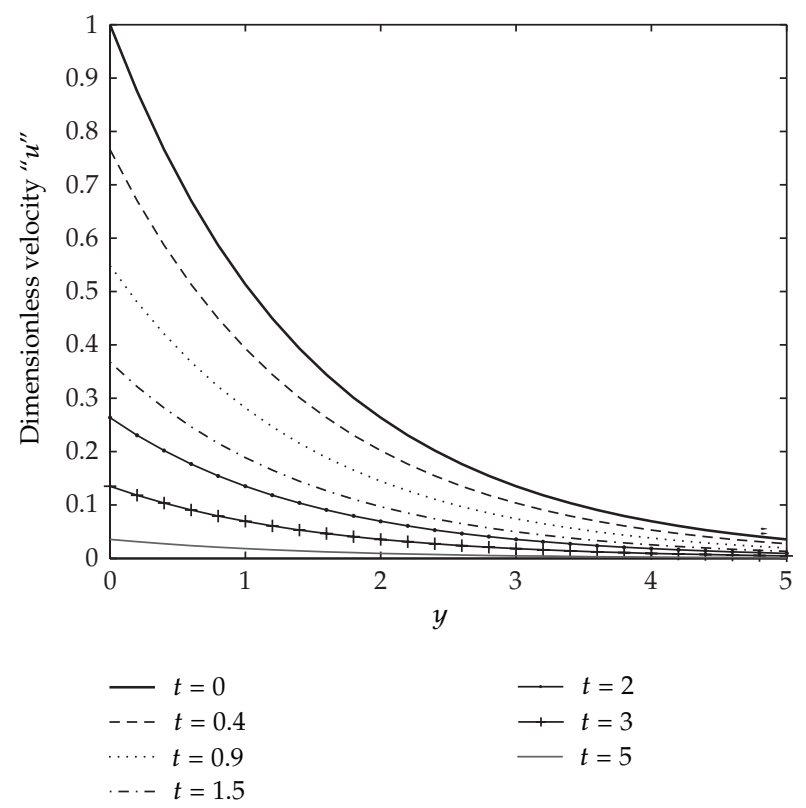

Figure 1: Backward wave-front type travelling wave solution (4.12) varying $t$ when $\gamma=0.5, \beta=2$, and $c=1$ are fixed.

The solution (4.12) satisfies the initial and boundary conditions (3.9) for the particular values of the unspecified functions $V(t), f(y), g(y)$, and $h(y)$. Using (4.12) in (3.9) results in

$$
\begin{gathered}
u(0, t)=V(t)=\exp \left(\frac{-\beta c t}{6 c \gamma}\right) \\
u(y, 0)=f(y)=\exp \left(\frac{-\beta y}{6 c \gamma}\right) \\
\frac{\partial u(y, 0)}{\partial t}=g(y)=\frac{-\beta}{6 \gamma} \exp \left(\frac{-\beta y}{6 c \gamma}\right) \\
\frac{\partial^{2} u(y, 0)}{\partial t^{2}}=h(y)=\frac{\beta^{2}}{(6 \gamma)^{2}} \exp \left(\frac{-\beta y}{6 c \gamma}\right) .
\end{gathered}
$$

Here $V(t), f(y), g(y)$, and $h(y)$ depend on the physical parameters of the flow. The solution (4.12) is plotted in Figures 1-4 for different values of the emerging parameters.

\subsection{Forward Wave-Front Type Travelling Wave Solutions}

We look for invariant solutions under the operator $X_{1}+c X_{2}$ (with $c>0$ ) which represent forward wave-front type travelling wave solutions with constant wave speed $c$. In this case, the waves are propagating away from the plate. These are solutions of the form

$$
u(y, t)=G(\eta) \text { with } \eta=y-c t
$$




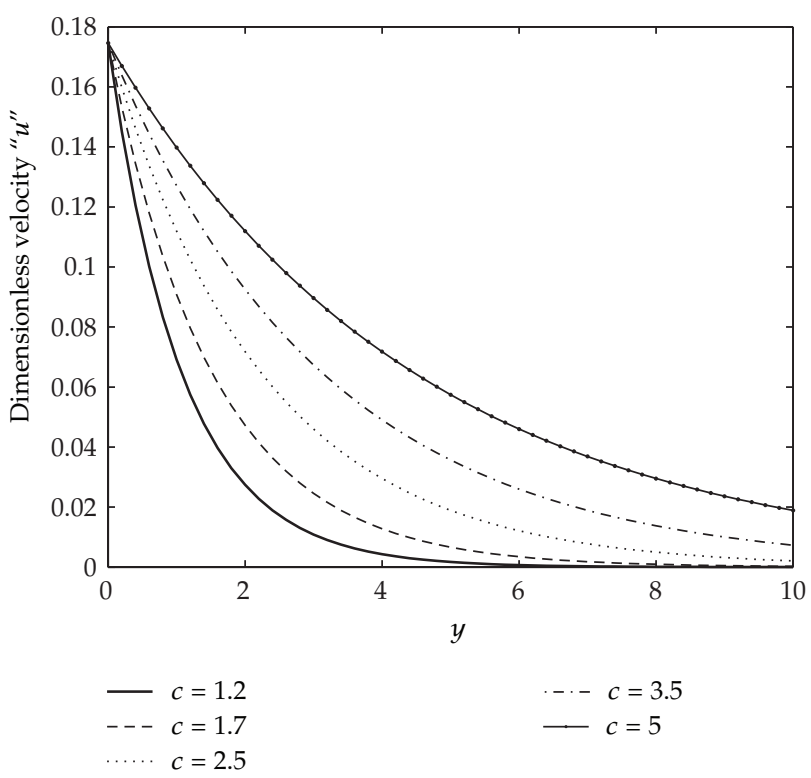

Figure 2: Backward wave-front type travelling wave solution (4.12) varying $c$ when $\gamma=0.6, \beta=4$, and $t=\pi / 2$ are fixed.

Using (4.14) in (3.8) results in a fifth-order ordinary differential equation for $G(\eta)$,

$$
-c \frac{d G}{d \eta}=\frac{d^{2} G}{d \eta^{2}}-\alpha c \frac{d^{3} G}{d \eta^{3}}+\beta_{1} c^{2} \frac{d^{4} G}{d \eta^{4}}+\beta\left(\frac{d G}{d \eta}\right)^{2} \frac{d^{2} G}{d \eta^{2}}-\gamma_{1} c^{3} \frac{d^{5} G}{d \eta^{5}}-2 \gamma \frac{d}{d \eta}\left[c\left(\frac{d G}{d \eta}\right)^{2} \frac{d^{2} G}{d \eta^{2}}\right] .
$$

Following the same methodology adopted for the backward wave-front type travelling wave solutions, the above equation admits exact solutions of the form

$$
G(\eta)=A \exp \left[\frac{\beta \eta}{6 c \gamma}\right]
$$

provided

$$
\frac{\beta}{6 \gamma}+\frac{\beta^{2}}{(6 c \gamma)^{2}}-\frac{\beta^{3} \alpha c}{(6 c \gamma)^{3}}+\frac{\beta_{1} \beta^{4} c^{2}}{(6 c \gamma)^{4}}-\frac{\gamma \beta^{5} c^{3}}{(6 c \gamma)^{5}}=0
$$

Thus, (3.8) subject to (4.17) admits the exact solution

$$
u(y, t)=\exp \left[\frac{\beta(y-c t)}{6 c r}\right] \text { with } c>0 .
$$




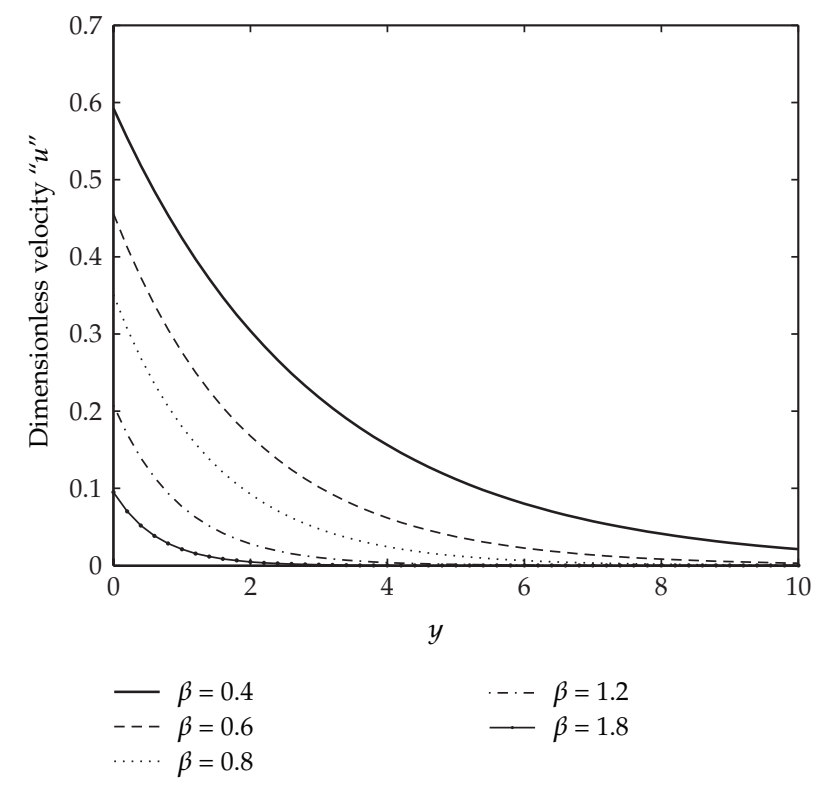

Figure 3: Backward wave-front type travelling wave solution (4.12) varying $\beta$ when $\gamma=0.2, c=1$, and $t=\pi / 2$ are fixed.

Note that the solution (4.18) does not satisfy the second boundary condition at infinity but satisfies the rest of the boundary conditions for the particular values of the functions $V(t)$, $f(y), g(y)$, and $h(y)$. Using (4.18) in (3.9) gives

$$
\begin{gathered}
u(0, t)=V(t)=\exp \left(\frac{-\beta c t}{6 c \gamma}\right), \\
u(y, 0)=f(y)=\exp \left(\frac{\beta y}{6 c \gamma}\right), \\
\frac{\partial u(y, 0)}{\partial t}=g(y)=\frac{-\beta}{6 \gamma} \exp \left(\frac{\beta y}{6 c \gamma}\right), \\
\frac{\partial^{2} u(y, 0)}{\partial t^{2}}=h(y)=\frac{\beta^{2}}{(6 r)^{2}} \exp \left(\frac{\beta y}{6 c \gamma}\right) .
\end{gathered}
$$

\section{Conditional Symmetry Solution}

We consider the PDE (3.8) with the invariant surface condition

$$
u_{t}=\lambda u,
$$




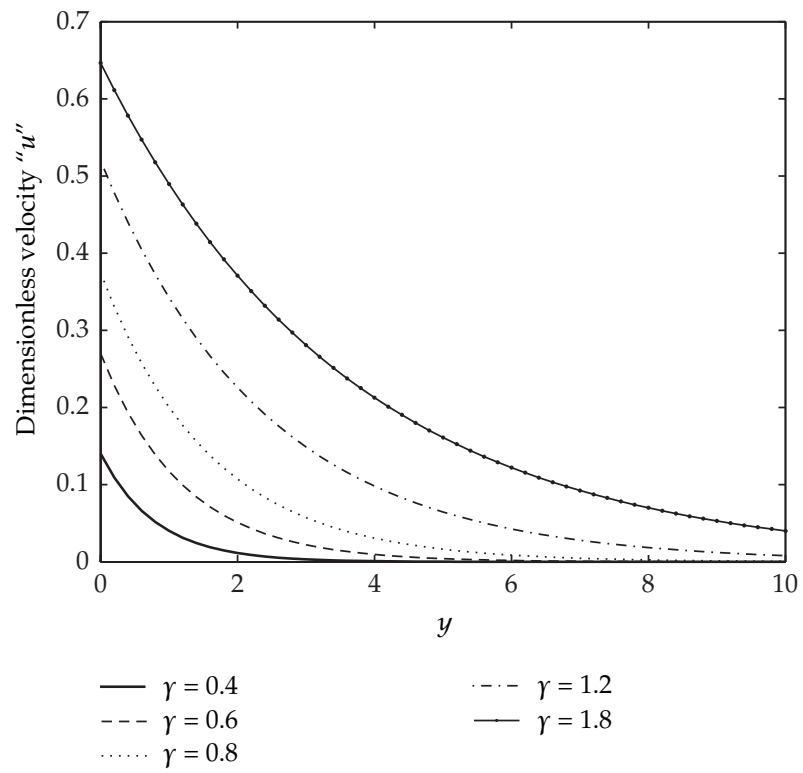

Figure 4: Backward wave-front type travelling wave solution (4.12) varying $\gamma$ when $\beta=3, c=1$, and $t=\pi / 2$ are fixed.

where $\lambda$ is a constant to be found. This corresponds to the operator

$$
X=\frac{\partial}{\partial t}+\lambda u \frac{\partial}{\partial u}
$$

The invariant solution corresponding to (5.2) is

$$
u(y, t)=\exp (\lambda t) F(y)
$$

where $F(y)$ is an undetermined function of $y$.

Substituting (5.3) in (3.8) leads to a linear second-order ordinary differential equation in $F(y)$, given by

$$
\frac{d^{2} F}{d y^{2}}-\left(\frac{\lambda}{1+\alpha \lambda+\beta_{1} \lambda^{2}+\gamma_{1} \lambda^{3}}\right) F=0 .
$$

and the relation

$$
\beta+6 \gamma \lambda=0
$$

From (5.5), we deduce

$$
\lambda=-\frac{\beta}{6 \gamma} \text {. }
$$


Journal of Applied Mathematics

Using the value of $\lambda$ in (5.4), we get

$$
\frac{d^{2} F}{d y^{2}}-\left\{\frac{(-\beta / 6 \gamma)}{\left(1-\alpha(\beta / 6 \gamma)+\beta_{1}(\beta / 6 \gamma)^{2}-\gamma_{1}(\beta / 6 \gamma)^{3}\right)}\right\} F=0,
$$

The reduced ODE (5.7) is solved subject to the boundary conditions

$$
\begin{gathered}
F(0)=1, \\
F(y) \longrightarrow 0 \text { as } y \longrightarrow \infty .
\end{gathered}
$$

Now we solve (5.7) subject to the boundary conditions (5.8) for three different cases.

Case $1\left(\left[(-\beta / 6 \gamma) /\left(1-\alpha(\beta / 6 \gamma)+\beta_{1}(\beta / 6 \gamma)^{2}-\gamma_{1}(\beta / 6 \gamma)^{3}\right)\right]=0\right)$. We have

$$
\frac{d^{2} F}{d y^{2}}=0
$$

Solution of (5.9) subject to the boundary conditions (5.8) is

$$
F(y)=\text { constant }(\text { say } C)
$$

In this case, the solution for $u(y, t)$ is

$$
u(t)=C \exp \left(-\frac{\beta}{6 \gamma} t\right)
$$

Thus, a time-dependent solution is obtained in this case.

Case $2\left(\left[(-\beta / 6 \gamma) /\left(1-\alpha(\beta / 6 \gamma)+\beta_{1}(\beta / 6 \gamma)^{2}-\gamma_{1}(\beta / 6 \gamma)^{3}\right)\right]<0\right)$. In this case, the solution for $F(y)$ is written as

$$
\begin{aligned}
F(y)= & a \cos \left[\sqrt{\left(\frac{(-\beta / 6 \gamma)}{1-\alpha(\beta / 6 \gamma)+\beta_{1}(\beta / 6 \gamma)^{2}-\gamma_{1}(\beta / 6 \gamma)^{3}}\right) y}\right] \\
& +b \sin \left[\sqrt{\left(\frac{(-\beta / 6 \gamma)}{1-\alpha(\beta / 6 \gamma)+\beta_{1}(\beta / 6 \gamma)^{2}-\gamma_{1}(\beta / 6 \gamma)^{3}}\right) y} .\right.
\end{aligned}
$$

Since $F(\infty)=0$, an unbounded solution is obtained for $F(y)$. Thus, the solution for $u(y, t)$ does not exist in this case. 
Case $3\left(\left[(-\beta / 6 \gamma) /\left(1-\alpha(\beta / 6 \gamma)+\beta_{1}(\beta / 6 \gamma)^{2}-\gamma_{1}(\beta / 6 \gamma)^{3}\right)\right]>0\right)$. In this case, the solution for $F(y)$ is given by

$$
\begin{aligned}
F(y)= & a \exp \left[\left\{\sqrt{\left(\frac{(-\beta / 6 \gamma)}{1-\alpha(\beta / 6 \gamma)+\beta_{1}(\beta / 6 \gamma)^{2}-\gamma_{1}(\beta / 6 \gamma)^{3}}\right)}\right\} y\right] \\
& +b \exp \left[-\left\{\sqrt{\left(\frac{(-\beta / 6 \gamma)}{1-\alpha(\beta / 6 \gamma)+\beta_{1}(\beta / 6 \gamma)^{2}-\gamma_{1}(\beta / 6 \gamma)^{3}}\right)}\right\} y\right],
\end{aligned}
$$

By applying the boundary conditions (5.8), we have $a=0$ (for a bounded solution) and $b=1$ (by using first the boundary condition). Thus, the solution for $F(y)$ is

$$
F(y)=\exp \left[-\left\{\sqrt{\left(\frac{(-\beta / 6 \gamma)}{1-\alpha(\beta / 6 \gamma)+\beta_{1}(\beta / 6 \gamma)^{2}-\gamma_{1}(\beta / 6 \gamma)^{3}}\right)}\right\} y\right] .
$$

Substituting $F(y)$ into (5.3), we obtain the solution for $u(y, t)$ in the form

$$
u(y, t)=\exp \left[-\left\{\left(\frac{\beta}{6 \gamma}\right) t+\sqrt{\left(\frac{(-\beta / 6 \gamma)}{1-\alpha(\beta / 6 \gamma)+\beta_{1}(\beta / 6 \gamma)^{2}-\gamma_{1}(\beta / 6 \gamma)^{3}}\right)} y\right\}\right] .
$$

Note that the conditional symmetry solution (5.15) satisfies the initial and the boundary conditions (3.9)-(21) with

$$
\begin{gathered}
V(t)=\exp \left[-\left(\frac{\beta}{6 \gamma}\right) t\right], \\
f(y)=\exp \left(-\sqrt{\left(\frac{(-\beta / 6 \gamma)}{1-\alpha(\beta / 6 \gamma)+\beta_{1}(\beta / 6 \gamma)^{2}-\gamma_{1}(\beta / 6 \gamma)^{3}}\right) y}\right), \\
g(y)=-\left(\frac{\beta}{6 \gamma}\right) \exp \left(-\sqrt{\left(\frac{(-\beta / 6 \gamma)}{1-\alpha(\beta / 6 \gamma)+\beta_{1}(\beta / 6 \gamma)^{2}-\gamma_{1}(\beta / 6 \gamma)^{3}}\right) y}\right), \\
h(y)=\left(\frac{\beta}{6 \gamma}\right)^{2} \exp \left(-\sqrt{\left(\frac{(-\beta / 6 \gamma)}{1-\alpha(\beta / 6 \gamma)+\beta_{1}(\beta / 6 \gamma)^{2}-\gamma_{1}(\beta / 6 \gamma)^{3}}\right) y}\right),
\end{gathered}
$$

where $V(t), f(y), g(y)$, and $h(y)$ depend on physical parameters of the flow. The graphical behavior of solution (5.15) is shown in Figure 5.

Remark 5.1. The backward wave-front type travelling wave closed-form solution (4.12) and the conditional symmetry solution (5.15) best represent the physics of the problem considered in the sense that these solutions satisfy all the initial and the boundary conditions 


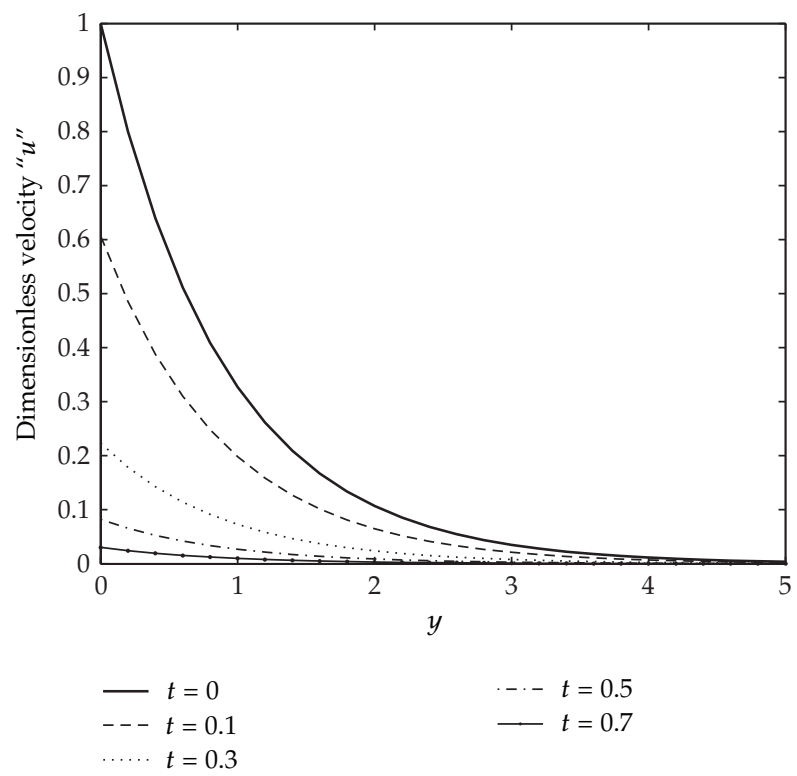

Figure 5: Conditional symmetry solution (5.15) varying $t$ when $\gamma=0.2, \gamma_{1}=0.1, \beta=6, \beta_{1}=0.5$, and $\alpha=1$ are fixed.

and also show the effects of different emerging parameters of the flow problem given in Figures 1-5. The forward wave-front type travelling wave solution (4.18) does not satisfy the second boundary condition at infinity. As a consequence, it does not show the behavior of the physical model. But this solution does show the shockwave behavior of the flow. To emphasize, we say that the forward wave-front type travelling wave solution is actually a shockwave solution with slope approaching infinity along the characteristic $\eta=y-c t$, as shown in Figure 6.

\section{Physical Interpretation of the Results}

In order to explain and understand the physical structure of the flow problem, various graphs are plotted in Figures 1-6.

Figure 1 shows the influence of time $t$ on the backward wave-front type travelling wave solution (4.12). This figure depicts that velocity decreases as time increases. Clearly, the variation of velocity is observed for $0 \leqslant t \leqslant 5$. For $t>5$, the velocity profile remains the same. In other words, we can say that the steady-state behavior of the velocity is achieved for $t>5$.

Figure 2 shows the effects of the wave speed $c$ on the velocity profile. It is clearly observed that with the increase of wave speed $c$, the velocity profile is increasing. So in this way, we can remark that both time $t$ and the wave speed $c$ have opposite effects on the backward wave-front type travelling wave solution (4.12) of the governing model.

Figures 3 and 4 have been plotted to see the influence of the third grade parameter $\beta$ and fourth grade parameter $\gamma$ on the backward wave-front type travelling wave solution (4.12) of the flow problem. These figures reveal that both $\beta$ and $\gamma$ have an opposite behavior on the structure of the velocity, that is, with an increase in the third grade parameter $\beta$, the velocity profile decreases showing the shear thickening behavior of the fluid, whereas 


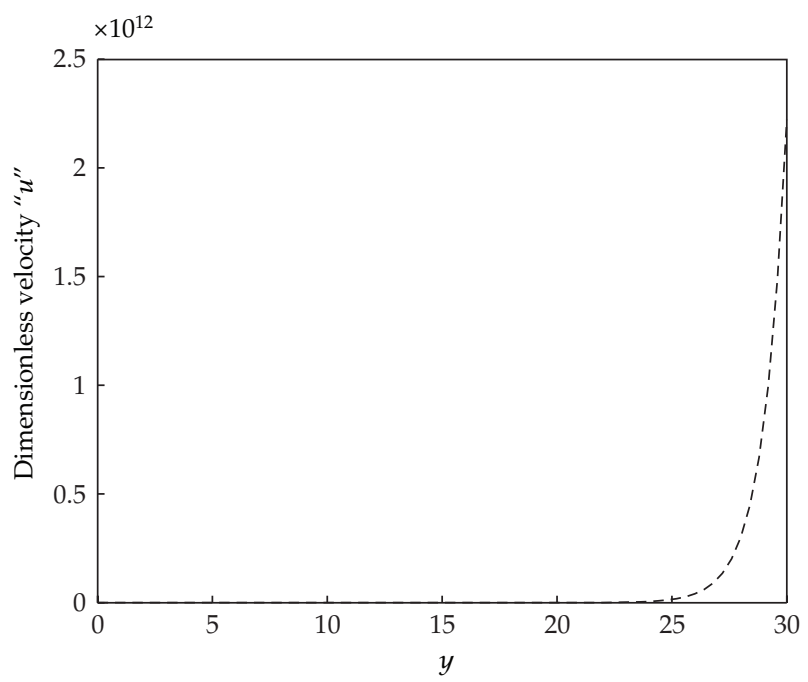

Figure 6: Plot of the forward wave-front type travelling wave solution (4.18) when $\gamma=0.5, \beta=3, c=1$, and $t=\pi / 2$ are fixed.

the velocity field increases for increasing values of the fourth grade parameter $\gamma$, which shows the shear thinning behavior of the model. This is in accordance with the fact that a fourth grade fluid model predicts both shear thickening and the shear thinning properties of the flow.

In Figure 5, the conditional symmetry solution (5.15) is plotted against the increasing values of time $t$. The behavior of time on the conditional symmetry solution is same as observed previously for the backward wave-front type travelling wave solution. That is, with the increase in time $t$, the velocity decreases. However, in this case, the steady-state behavior of the velocity is achieved quicker as compared to the backward wave-front type travelling wave solution. The variation of velocity is observed for $0 \leqslant t \leqslant 0.7$. For $t>0.7$, and the steady-state behavior of the velocity is observed.

In Figure 6, the forward wave-front type travelling wave solution (4.18) is plotted. This figure describes the shock wave behavior of the flow with slope approaching infinity along the characteristic. This solution does not show the physics of the model but does predict the hidden shock wave phenomena in the flow. Some examples of shock waves are moving shock, detonation wave, detached shock, attached shock, recompression shock, shock in a pipe flow, shock waves in rapid granular flows, shock waves in astrophysics, and so on.

\section{Concluding Remarks}

The present work has been undertaken in order to investigate further the regime of a fourth grade non-Newtonian fluid model. Some reductions and exact (closed-form) solutions for the time-dependent flow of a fourth grade fluid have been established using the symmetry approach. Travelling wave and conditional symmetry solutions are obtained for the governing nonlinear PDE. Both forward and the backward wave-front type travelling wave solutions have been constructed. The better solutions from the physical point of view of the model considered are the conditional symmetry solution and the backward wave-front type travelling wave solution. This issue has also been addressed in detail in Remark 5.1. 
Moreover, the work contained herein is theoretical in nature and is a prototype model. The methods used will be helpful for a wide range of nonlinear problems in fluids given the paucity of known exact solutions especially in non-Newtonian fluids.

\section{Acknowledgments}

T. Aziz thanks the University of the Witwatersrand for the scholarship. The author also thanks the organizers of the conference SDEA 2012 for their warm hospitality.

\section{References}

[1] W. Tan and T. Masuoka, "Stokes' first problem for an Oldroyd-B fluid in a porous half space," Physics of Fluids, vol. 17, no. 2, Article ID 023101, 7 pages, 2005.

[2] C. Fetecau, S. C. Prasad, and K. R. Rajagopal, "A note on the flow induced by a constantly accelerating plate in an Oldroyd-B fluid," Applied Mathematical Modelling, vol. 31, no. 4, pp. 647-654, 2007.

[3] T. Hayat, R. J. Moitsheki, and S. Abelman, "Stokes' first problem for Sisko fluid over a porous wall," Applied Mathematics and Computation, vol. 217, no. 2, pp. 622-628, 2010.

[4] M. Molati, T. Hayat, and F. Mahomed, "Rayleigh problem for a MHD Sisko fluid," Nonlinear Analysis: Real World Applications, vol. 10, no. 6, pp. 3428-3434, 2009.

[5] P. D. Ariel, "Flow of a third grade fluid through a porous flat channel," International Journal of Engineering Science, vol. 41, no. 11, pp. 1267-1285, 2003.

[6] Y. Aksoy and M. Pakdemirli, "Approximate analytical solutions for flow of a third-grade fluid through a parallel-plate channel filled with a porous medium," Transport in Porous Media, vol. 83, no. 2, pp. 375-395, 2010.

[7] F. T. Akyildiz, H. Bellout, and K. Vajravelu, "Exact solutions of nonlinear differential equations arising in third grade fluid flows," International Journal of Non-Linear Mechanics, vol. 39, no. 10, pp. 1571-1578, 2004.

[8] M. B. Akgül and M. Pakdemirli, "Analytical and numerical solutions of electro-osmotically driven flow of a third grade fluid between micro-parallel plates," International Journal of Non-Linear Mechanics, vol. 43, no. 9, pp. 985-992, 2008.

[9] G. Saccomandi, "Group properties and invariant solutions of plane micropolar flows," International Journal of Engineering Science, vol. 29, no. 5, pp. 645-648, 1991.

[10] A. Aziz and T. Aziz, "MHD flow of a third grade fluid in a porous half space with plate suction or injection: an analytical approach," Applied Mathematics and Computation, vol. 218, no. 21, pp. 1044310453, 2012.

[11] T. Aziz, F. M. Mahomed, and A. Aziz, "Group invariant solutions for the unsteady MHD flow of a third grade fluid in a porous medium," International Journal of Non-Linear Mechanics, vol. 47, no. 7, pp. 792-798, 2012.

[12] T. Hayat, H. Mambili-Mamboundou, C. M. Khalique, and F. M. Mahomed, "Effect of magnetic field on the flow of a fourth order fluid," Nonlinear Analysis: Real World Applications, vol. 10, no. 6, pp. 3413-3419, 2009.

[13] T. Hayat, A. H. Kara, and E. Momoniat, “The unsteady flow of a fourth-grade fluid past a porous plate," Mathematical and Computer Modelling, vol. 41, no. 11-12, pp. 1347-1353, 2005.

[14] W. R. Schowalter, Mechanics of Non-Newtonian Fluids, Pergamon, New York, NY, USA, 1978.

[15] Y. Wang and W. Wu, "Unsteady flow of a fourth-grade fluid due to an oscillating plate," International Journal of Non-Linear Mechanics, vol. 42, no. 3, pp. 432-441, 2007.

[16] A. M. Siddiqui, R. Mahmood, and Q. K. Ghori, "Homotopy perturbation method for thin film flow of a fourth grade fluid down a vertical cylinder," Physics Letters, Section A, vol. 352, no. 4-5, pp. 404-410, 2006.

[17] M. Sajid, T. Hayat, and S. Asghar, "On the analytic solution of the steady flow of a fourth grade fluid," Physics Letters, Section A, vol. 355, no. 1, pp. 18-26, 2006. 
[18] T. Hayat, M. Sajid, and M. Ayub, "On explicit analytic solution for MHD pipe flow of a fourth grade fluid," Communications in Nonlinear Science and Numerical Simulation, vol. 13, no. 4, pp. 745-751, 2008.

[19] S. Nadeem, T. Hayat, S. Abbasbandy, and M. Ali, "Effects of partial slip on a fourth-grade fluid with variable viscosity: an analytic solution," Nonlinear Analysis: Real World Applications, vol. 11, no. 2, pp. 856-868, 2010.

[20] T. Hayat and S. Noreen, "Peristaltic transport of fourth grade fluid with heat transfer and induced magnetic field," C. R. Mecanique, vol. 338, no. 9, pp. 518-528, 2010.

[21] V. Marinca, N. Herişanu, C. Bota, and B. Marinca, "An optimal homotopy asymptotic method applied to the steady flow of a fourth-grade fluid past a porous plate," Applied Mathematics Letters, vol. 22, no. 2, pp. 245-251, 2009.

[22] L. V. Ovsiannikov, Group Analysis of Differential Equations, Academic Press, New York, NY, USA, 1982.

[23] P. J. Olver, Applications of Lie Groups to Differential Equations, vol. 107 of Graduate Texts in Mathematics, Springer, New York, NY, USA, 1986.

[24] C. Wafo Soh, "Invariant solutions of the unidirectional flow of an electrically charged power-law non-Newtonian fluid over a flat plate in presence of a transverse magnetic field," Communications in Nonlinear Science and Numerical Simulation, vol. 10, no. 5, pp. 537-548, 2005.

[25] P. Y. Picard, "Some exact solutions of the ideal MHD equations through symmetry reduction method," Journal of Mathematical Analysis and Applications, vol. 337, no. 1, pp. 360-385, 2008.

[26] M. Pakdemirli, M. Yürüsoy, and A. Küçükbursa, "Symmetry groups of boundary layer equations of a class of non-Newtonian fluids," International Journal of Non-Linear Mechanics, vol. 31, no. 3, pp. 267-276, 1996.

[27] M. Yürüsoy, "Similarity solutions for creeping flow and heat transfer in second grade fluids," International Journal of Non-Linear Mechanics, vol. 39, no. 4, pp. 665-672, 2004.

[28] M. Yürüsoy, "Unsteady boundary layer flow of power-law fluid on stretching sheet surface," International Journal of Engineering Science, vol. 44, no. 5-6, pp. 325-332, 2006.

[29] G. W. Bluman and J. D. Cole, “The general similarity solution of the heat equation," vol. 18, pp. 10251042, 1969.

[30] D. J. Arrigo and J. M. Hill, “Nonclassical symmetries for nonlinear diffusion and absorption," Studies in Applied Mathematics, vol. 94, no. 1, pp. 21-39, 1995.

[31] S. Spichak and V. Stognii, "Conditional symmetry and exact solutions of the Kramers equation," in Symmetry in Nonlinear Mathematical Physics, vol. 1-2, pp. 450-454, National Academy of Sciences of Ukraine. Institute of Mathematics, Kiev, Ukraine, 1997.

[32] A. F. Barannyk and Yu. D. Moskalenko, "Conditional symmetry and exact solutions of the multidimensional nonlinear d'Alembert equation," Journal of Nonlinear Mathematical Physics, vol. 3, no. 3-4, pp. 336-340, 1996. 


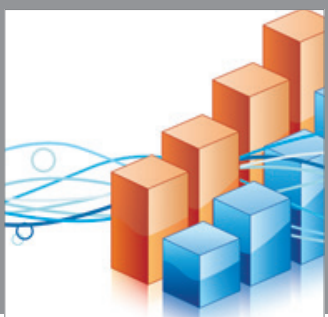

Advances in

Operations Research

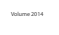

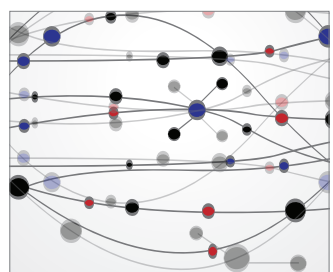

\section{The Scientific} World Journal
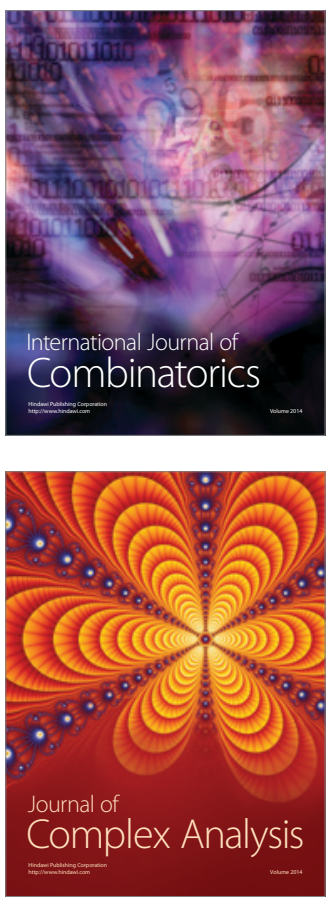

International Journal of

Mathematics and

Mathematical

Sciences
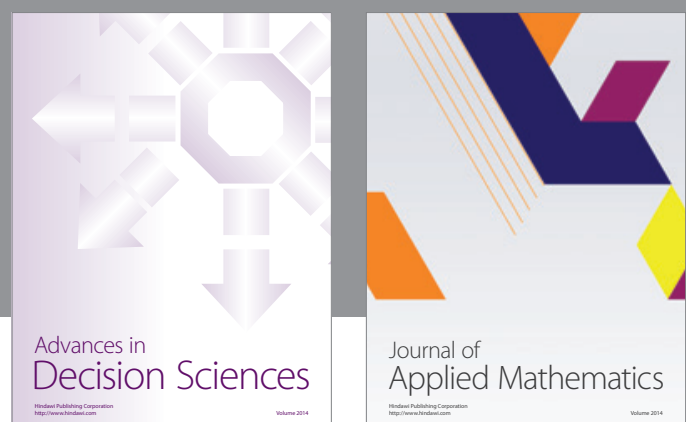

Journal of

Applied Mathematics
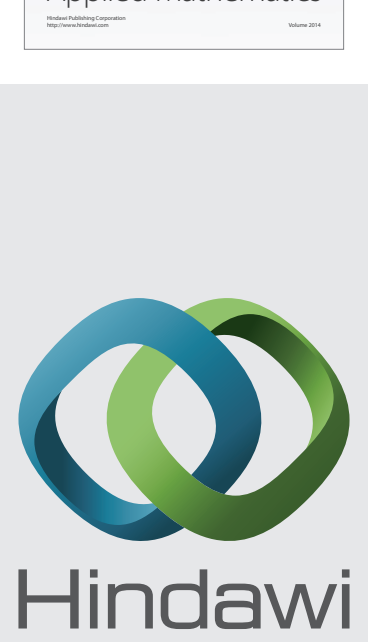

Submit your manuscripts at http://www.hindawi.com
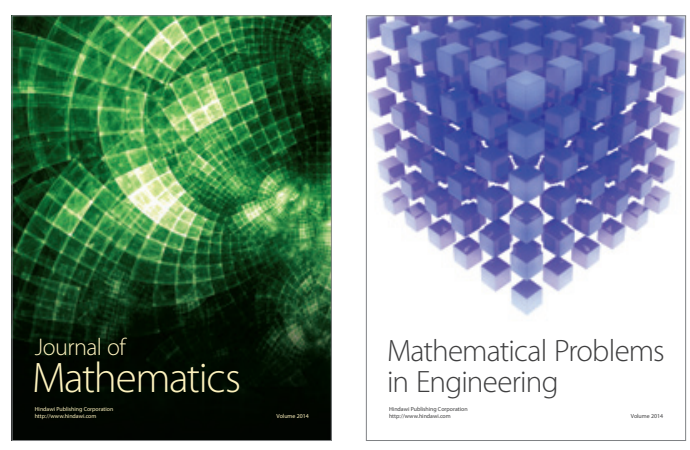

Mathematical Problems in Engineering
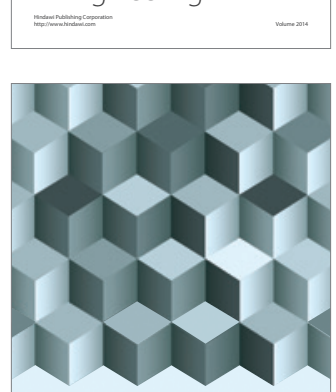

Journal of

Function Spaces
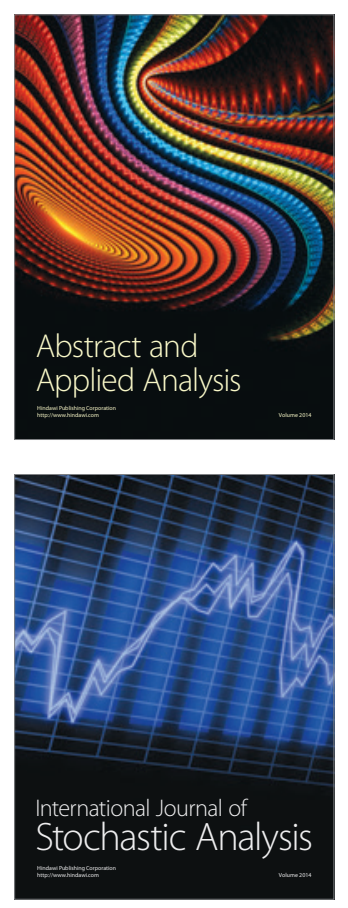

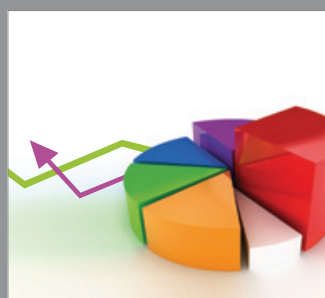

ournal of

Probability and Statistics

Promensencen
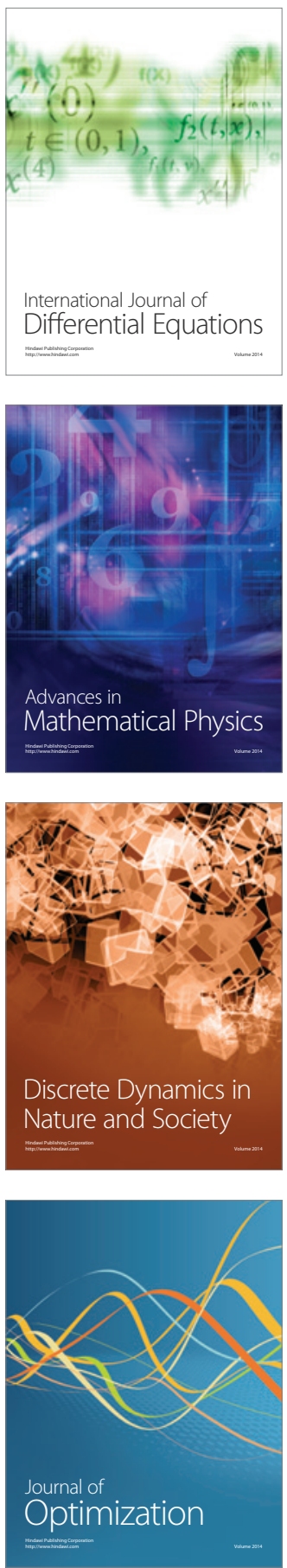